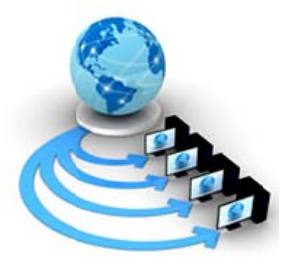

Volume 9, No. 1, January-February 2018

International Journal of Advanced Research in Computer Science

RESEARCH PAPER

\title{
AECHE: ADAPTIVE ENERGY EFFICIENT CLUSTER HEAD ELECTION MECHANISM FOR REACTIVE WIRELESS SENSOR NETWORKS
}

\author{
Sundareswaran P \\ Department of Computer Science and Engineering, \\ Manonmaniam Sundaranar University, \\ Tirunelveli,627012, India
}

\author{
Rajesh R S \\ Department of Computer Science and Engineering, \\ Manonmaniam Sundaranar University, \\ Tirunelveli,627012, India
}

\begin{abstract}
Wireless sensor network (WSN) has developed as one of the most promising technologies for the future. This has been achieved by advances in technology to avail small, inexpensive, and smart sensors, which result in cost effective and easily deployable WSNs. Clustering technique is used in communication protocols, so that considerable amount of energy has been conserved while transmitting the data to the cluster head rather than directly to the far away sink. In some applications, the sensors will be triggered to send data when the sensed data exceed the threshold value. In these circumstances, limited number of sensor nodes gets activated and sends the data and remaining nodes are under idle mode. Hence energy consumption is more in these activated regions, which leads to death of sensors nodes. It is concentrated on these activated sensors and find the change in the ratio between the active nodes and alive nodes to calculate the optimal cluster number for each round. The optimum number of clusters is calculated so that the balanced clusters are created. Extensive analysis has been made with existing protocols, and found that the proposed methodology out performs the existing protocol in terms of networks life time and throughput.
\end{abstract}

Keywords: Wireless Sensor Network;TEEN, Routing; Clustering Protocol,Energy

\section{INTRODUCTION}

A Wireless Sensor Network (WSN) is a distributed network consists of a large number of distributed, selfdirected, tiny, low powered, limited process capacity devices called sensor nodes alias motes. The applications like forest fire detection, transportation, weather forecasting, environment monitoring, battle field surveillance, health monitoring and IoT are using the role of wireless Sensor Networks (WSNs).[1-8]

As the sensor nodes are low cost and having sensing capability, they are widely used in the common applications used by humans. The large numbers of tiny, energy constrained sensors are inter-connected together to form a wireless sensor network. The sensors can sense analog information from the environment and digitize it before sending to the sink or to the base station.

The issues while deploying the sensor nodes are due to coverage, energy, localization, and data security. The key issue among them is energy, since the nodes are provided with limited battery power and mostly not changeable. Since battery cannot be recharged in most of the applications as being placed in critical locations, it is hard and even not possible to change or recharge batteries of sensor nodes. Therefore the minimum consumption of energy will increase the life time of the nodes, which increases the entire lifetime of WSN.. Energy consumption is occurred in a node while data are transmitted. In order to prolong the network life time, a lot of energy conservation techniques are applied in WSNs [16]. Data aggregation technique is applied to reduce the transmission by removing the redundant data [9-10].

The most common technique to minimize data communications among sensor nodes is clustering. In general, all the sensor nodes in a sensor network send their data directly to the sink. It leads to increase in network overhead and the network lifetime is decreased. Therefore clustering technique has been used in WSNs. The entire sensor network has been divided into number of disjoint sets called clusters and each cluster has its own cluster head. The nodes belong to the cluster send data to their cluster head rather than sending directly to the far away sink. The network overhead in considerably reduced and energy of each node is preserved when clustering concept is used.

A lot of clustering algorithms have been developed for WSNs to prolong the network life time [12,16-18,22-24] WSNs have been classified into two categories viz. homogeneous and heterogeneous WSNs. In homogeneous WSNs, the initial energy level of each node in a sensor network is identical for each node. On the other hand the heterogeneous WNSs have sensor nodes with different energy levels and different attributes. Premier protocols like LEACH [18], TEEN [23], PEGASIS [22] are the examples of clustering protocols used in homogeneous WSNs. SEP [12], DEEC [20] are the clustering protocols used for heterogeneous WSNs. Most of the clustering protocols basically have the feature of data aggregation so that the redundant data have been eliminated from transmission which also preserves energy $[11,13]$.

Most of the clustering algorithms has two phases: set up phase and steady state phase. The clusters are formed and cluster heads are elected in set up phase and data are sensed and transmitted in steady state phase as per the TDMA schedule sent by the cluster head to its cluster members.

Depending on the function and application, the WSNs are categorized into two group viz. reactive and proactive networks. The sensors used in proactive sensor network sense the environment at specific intervals and broadcast the data to the sink. Therefore the sensors and transmitters are always on in this case and send the parameters at regular intervals. These types of WSNs are mostly suitable for 
periodic monitoring applications. In the reactive networks, the nodes trigger immediately to rapid and sweeping deviation in the value of the sensed attribute. Therefore they are mostly suitable for time critical applications. Majority of clustering protocols fix the number of clusters based on the probability ratio which is decided at the start of functioning of the sensor network, so that the cluster count is constant. In this proposed model, an exponential function is used to derive the cluster count from the number of active nodes and the number of alive nodes in the network. This exponential function gives the optimum number of clusters at each round so that energy consumption is reduced considerably and the lifetime of the network is increased. The remaining part of the paper is organized as follows. Related works are discussed in section 2, section 3 describes the proposed methodology, simulation results are discussed in section 4 and section 5 concludes the paper.

\section{RELATED WORK}

As energy is an important criterion for the better function of Wireless Sensor Nodes (WSNs), most of the protocols used in sensor networks concentrate on energy conservation of sensor nodes. The clustering protocols used in sensor networks also have the primary attention in saving the energy of the nodes. One of the premier protocols used in Wireless Sensor Networks (WSNs) is LEACH [18]. The Low Energy Adaptive Clustering Hierarchy Protocol uses randomized rotation of cluster heads among the nodes, which effectively decreases the usage of battery energy of each node, in turn increases overall energy level of the WSN. The data aggregation and fusion also play a major role in reducing the energy consumption when using LEACH. The redundant data will be combined to a single data and that will be sent to the base station or sink by the cluster heads, so that multiple transmissions of same data are reduced. The sensor nodes elect $\mathrm{CHs}$ from $n$ nodes at regular time interval with a given probability. The probability threshold function to elect a cluster head is defined as

$T(n)=\left\{\begin{array}{l}\frac{p}{1-p *\left(\operatorname{rmod} \frac{1}{p}\right)} \text { if } n \varepsilon C \\ 0 \quad \text { Otherwise }\end{array}\right.$

Where $\mathrm{P}$ is the probability function of the nodes, $\mathrm{n}$ is the total number of sensor nodes present in the network, and $\mathrm{r}$ is the number of rounds. ( $\mathrm{r} \bmod 1 / \mathrm{P})$ is the number of nodes elected as cluster head in a cycle, and $C$ is the set of sensors not elected as a cluster head in recent rounds. Nodes with randomly generated value less than probability threshold value T(n) will only be selected as cluster head. In LEACH, the cluster count is depends on the probability ratio in each round.

Threshold Sensitive Energy Efficient Sensor Network (TEEN) [23] is one of the protocols used for reactive networks. At every change of cluster cycle, apart from the element to be sensed, the cluster head transmit the following values to its cluster members.

Hard Threshold: This is the threshold value for the attribute to be sensed, beyond which, if the sensed value is arrived, the node transmit the sensed data immediately to its cluster head.
Soft Threshold: This is the maximum variation between the last sensed value and present value, beyond which the node triggers to send the data to the cluster head.

In a sensor network having thousands of nodes, not all the nodes are get activated simultaneously. Certain percentage of nodes may be in the idle stage in a particular place due to the similar sensed data and there is the possibility of nodes getting activated in other place owing to the variation in the sensed data. In TEEN also similar to LEACH, the cluster count is based on the probability ratio at each round.

A three way message exchange is performed in [17] among the sensor node with its neighbors to elect the cluster head. A node will be elected as cluster head based on its residual energy and its degree.

Intra-cluster communication cost is also taken into consideration for selecting the cluster head in each round as in [16]. In HEED, proposed by Younis and Fahmy, along with this parameter, the node's residual energy is calculated for each round before electing the cluster head. The probability to become a cluster head for a node is

$$
C H_{\text {Prob }}=C_{\text {prob }} \frac{E_{\text {residual }}}{E_{\text {max }}}
$$

where $\mathrm{C}_{\text {prob }}$ is the optimal percentage of $\mathrm{CH}$ (Cluster Head) among all nodes in a network, $\mathrm{E}_{\text {residual }}$ is the estimated current energy of the node and $\mathrm{E}_{\max }$ is a reference maximum energy which is equal for all the nodes in the network. $\mathrm{CH}_{\text {prob }}$ cannot be allowed to fall below a threshold value. There are two types of status a node could send to its neighbors: viz. tentative status and final status. If the $\mathrm{CH}_{\text {prob }}$ value of a node is less than 1 , the node becomes a tentative $\mathrm{CH}$ and this node is converted to a normal node at a later iteration if it finds low cost $\mathrm{CH}$. If the $\mathrm{CH}_{\text {prob }}$ has reached 1 , the corresponding node permanently becomes a $\mathrm{CH}$. A chain based construction has been proposed in PEGASIS [22] from the farthest node to the closest node of the sink using the greedy algorithm. The data sensed by the far away node sends the data to the neighbor, which fuses the received data with its own one and send to the neighbor. This process is repeated until the data reached the destination. The leader node acts as the cluster head here.

The initial energy and residual energy levels are the prime factors influence in the selection of cluster heads in DEEC (Distributed Energy Efficient Clustering algorithm) [20]. There are two types of nodes used in these type of the network. The advance nodes are having more energy than the normal nodes by $\alpha$ time. The reference energy that a node should spend in each round can be calculated with the help of the network life time. Let $\mathrm{p}_{\mathrm{i}}=1 / \mathrm{n}_{\mathrm{i}}$, which can be also regarded as average probability to be a cluster-head during ni rounds. Let $E_{i}(r)$ denotes the energy of a node $i$ at round $r$. A weight is added with the probability value for the cluster head for heterogeneous nodes.

The value of probability ratio $\left(p_{i}\right)$ to be a cluster head for the current round is

$$
P_{i}=\left\{\begin{array}{cl}
\frac{P_{o p t} E_{i}(r)}{(1+\alpha m) \bar{E}(r)} & \text { if } S_{i} \text { is the normal node } \\
\frac{P_{o p t}(1+\alpha) E_{i}(r)}{(1+\alpha m) \bar{E}(r)} & \text { if } S_{i} \text { is the advanced node }
\end{array}\right.
$$


Where $\mathrm{E}(\mathrm{r})$ is the average energy of the network at round $r$.

This $p_{i}$ can be used in calculating the probability threshold as

$T\left(S_{i}\right)=\left\{\begin{array}{l}\frac{P_{i}}{1-P_{i}\left(\bmod \frac{1}{P_{i}}\right)} \\ 0 \quad \text { Otherwise }\end{array}\right.$ if $S_{i} \varepsilon M$

where $M$ is the set of eligible nodes to be the cluster head at the round $r$. In DEEC, the energy of the nodes in each cluster in evenly depleted so that the cluster head is also distributed evenly. The cluster heads elected using the popular protocols are sometimes closely placed each other. This will cause increase in communication overhead and the network life time is decreased. This problem has been solved in [19]. Since this paper focus on the reactive sensor networks, the number nodes getting activated are depending on the threshold values. Therefore, the number of clusters is decided based on the change in number of nodes activated.

\section{PROPOSED METHODOLOGY}

\subsection{Network model}

Homogeneous wireless sensor network is considered for this work. There is no physical or hardware difference between cluster head and cluster member in this simulation environment. All the sensor nodes in this network model are having similar properties for the entire network and some sensor nodes will be acting as cluster head depending on the value of probability ratio. The first order radio model is considered for the simulation [21]. The first order radio model can be classified into free space model and multi path fading model based on the distance between the source and destination. Let $d_{o}$ be the threshold value for deciding the energy used for sending the data among the two models. The $d_{o}$ can be calculated as

$$
d_{0}=\sqrt{\frac{E_{f s}}{E_{m p}}}
$$

Where $E_{f s}$ and $E_{m p}$ are amplifier types in free space and multipath fading model respectively.

\section{Transmitting energy:}

Let $\mathrm{E}_{\mathrm{TX}}$ be the energy required for sending a packet of size $\mathrm{K}$ for a distance $d$. The energy $\mathrm{E}_{\mathrm{TX}}$ is calculated based on the distance value $d$ and $d_{o}$. If $d \leq d_{o}$, the free space model is used and for the other case the multi path fading model can be applied.

If $d \leq d_{o}$ then $\mathrm{E}_{\mathrm{TX}}=\left(\mathrm{E}_{\mathrm{elec}} * \mathrm{~K}\right)+\left(\mathrm{E}_{\mathrm{fs}} * \mathrm{~K} * d^{2}\right)$

If $d>d_{o}$ then $\mathrm{E}_{\mathrm{TX}}=\left(\mathrm{E}_{\mathrm{elec}} * \mathrm{~K}\right)+\left(\mathrm{E}_{\mathrm{mp}} * \mathrm{~K} * d^{4}\right)$

Where $E_{\text {elec }}=E_{t x}+E_{A G}$. In the case of cluster head, , $E_{A G}$ is the energy spent for data aggregation and for the normal sensor nodes $E_{A G}$ will be zero. The value $E_{\text {elec }}$ is the energy used for sending a bit $/ \mathrm{m} 2$.

\section{Receiving energy:}

Let $\mathrm{E}_{\mathrm{RC}}$ be the energy needed for receiving a packet of size $\mathrm{K}$ for a distance $d$.

$$
\mathrm{E}_{\mathrm{RC}}=\left(\mathrm{E}_{\mathrm{rx}} * \mathrm{~K}\right)
$$

Where $\mathrm{E}_{\mathrm{rx}}$ is the energy required for receiving a bit $/ \mathrm{m} 2$.

\subsection{AECHE Methodology}

In the reactive sensor networks, the nodes are activated only the sensed value exceeds the defined threshold values. In general, a wireless sensor network consists of large number of nodes and only a limited number of the nodes are activated due to the change in sensed value. In applications like fire detection and temperature monitoring, only limited number of the sensor nodes get triggered to send data due to surpassing of the sensed parameter beyond the threshold values. At that time, the remaining nodes are in idle state to preserve the energy. Therefore it is observed that the probability ratio $p_{o}$ for deciding the number of clusters used in earlier protocols is not feasible to compute optimum number of clusters. The ratio of number of active nodes to the total number of alive nodes is measured between consecutive rounds is computed. Let $m_{i}$ be the total number of active nodes at round $i$ and $n_{i}$ represents the total number of live nodes in the network at the round $i$, then

$$
\begin{aligned}
& x=\frac{m_{i}-m_{i-1}}{n_{i}-n_{i-1}} \\
& k=1-\frac{1}{e^{0.6 x}} \\
& \quad \text { Let } C H_{c n t} \text { represents the new cluster head count, then } \\
& \qquad H_{c n t}=p_{0} * n_{i} * k
\end{aligned}
$$

The probability ratio for the current round is replaced by the $p_{i}$ value. If the number of cluster heads calculated is less than the number of already selected cluster heads, the difference number of cluster heads will be changed as normal nodes. On the other hand, if the optimum number of cluster head is greater than the number of already selected cluster heads, the balance number of cluster heads is generated by using the same procedure adopted earlier to generate cluster heads. Then the clustering process in initiated and the clusters are created following the algorithm used in TEEN.

\section{Pseudo code for the cluster computation \\ $m \leftarrow$ total number of active nodes \\ $n \leftarrow$ total number of alive nodes \\ begin}

$X \leftarrow\left(\left(m_{i}-m_{i-1}\right) /\left(n_{i}-n_{i-1}\right)\right) * 10 * /$ calculating the ratio between active nodes and alive nodes */

$Y \leftarrow 1-e^{(-0.6 x)} \quad *$ / calculating the exponential value

corresponding to $x *$ /

$c h_{\text {cnt }} \leftarrow\left(\operatorname{ceil}\left(p_{o}{ }^{*} n_{i}{ }^{*} y\right) * /\right.$ finding the new cluster count $* /$

if $c h_{\text {cnt }}<p_{o}$

$p_{\text {wgt }} \leftarrow p_{o}-c h_{\text {cnt }}$

$p_{i} \leftarrow p_{o}-p_{w g t}$

else

$p_{\text {wgt }} \leftarrow c h_{\text {cnt }}-p_{o}$

$p_{i} \leftarrow p_{o}+p_{w g t}$

endif

if cluster $>c h_{\text {cnt }}$

for $c=1$ toch $_{\text {cnt }}$

for $a c h=1$ to (cluster- $(c-1)-1)$

ifnode(cluster(ach).id).energy<node(cluster(ach+1).id).ener

gy

Endif swap their ids. 
end

end

else

ifnode.energy $>0$

if rand $<=\left(p /\left(1-p^{*} \bmod (r\right.\right.$,round $\left.\left.(1 / p))\right)\right)$

cluster $=$ cluster +1

endif

node.type $=C$

endif

endif

\section{SIMULATION RESULTS}

The TEEN extension has been used for this simulation and the simulation has been performed in MATLAB R2013a. Modifications are made on the available TEEN extension to implement this methodology. Number of experiments is performed to compare the existing TEEN protocol with the proposed methodology. Experimental results show that the network life time and throughput are increased when compared with the existing TEEN protocol. Simulation environment with field dimension of $100 \mathrm{~m} \mathrm{x}$ $100 \mathrm{~m}$ has been considered for the experiment. The total number of sensor nodes deployed in the network is 500. The network parameters of the sensor network are given in Table 1.

Table 1. Network Parameters used in Simulation

\begin{tabular}{|c|c|}
\hline Parameters & Values \\
\hline Sink Position & $50 * 50$ \\
\hline $\mathrm{E}_{\mathrm{fs}}($ Amplifier used in free space model) & $10^{*} 10^{-12} \mathrm{j}$ \\
\hline $\begin{array}{l}\mathrm{E}_{\mathrm{mp}}(\text { Amplifier used in multipath fading } \\
\text { model) }\end{array}$ & $\begin{array}{l}0.0013 * 10 \\
12 \mathrm{j}\end{array}$ \\
\hline $\mathrm{E}_{\mathrm{AG}}$ (Aggregation Energy) & $5 * 10^{-9} \mathrm{j}$ \\
\hline Initial Energy $E_{0}$ & $0.01 \mathrm{j}$ \\
\hline Packet Length & 2000bits \\
\hline Control Packet Length & 100bits \\
\hline Probability of CHs $\left(\mathrm{P}_{\mathrm{o}}\right)$ & 0.1 \\
\hline Maximum rounds & 150 \\
\hline No of Nodes(n) & 500 \\
\hline
\end{tabular}

\subsection{Performance metrics}

The following metrics are selected to measure the performance of the AECHE methodology with the existing TEEN protocol.

\section{A) Network Life time}

It is the time duration between the starting time of the sensing and sending data by sensor nodes (beginning of the network) and the death of the last live node.

B) Throughput

It is the total amount of data received by the base station from the sensor nodes in a WSN.

\section{C) Stability period}

Stability period is the time or rounds between the start of the network and death of the first node.

Number of iterations is performed to find the optimum value as 0.6 in the expression

$1-\mathrm{e}^{-0.6 \mathrm{x}}$, so that a good result has been received. This equation is generalized during the problem analysis stage as $1-\mathrm{e}^{-\infty x}$. Iterations are performed with different values for $\infty$ ranging from 0.1 to 0.9 to find the optimum value which gives the best result.. Figure 1 show the lifetime analysis of the network. It indicates that the number of nodes alive is more after 80 rounds while using AECHE protocol than using TEEN protocol.

Figure 2 show the cluster count of the network in each round during simulation. The optimum number of clusters is selected when using the AECHE protocol. Therefore the network works in a better way even in critical situations. The number of clusters will be calculated in each round of operation based on the probability ratio $p_{o}$ and the new value $p_{i}$ will be assigned to $p_{o}$.

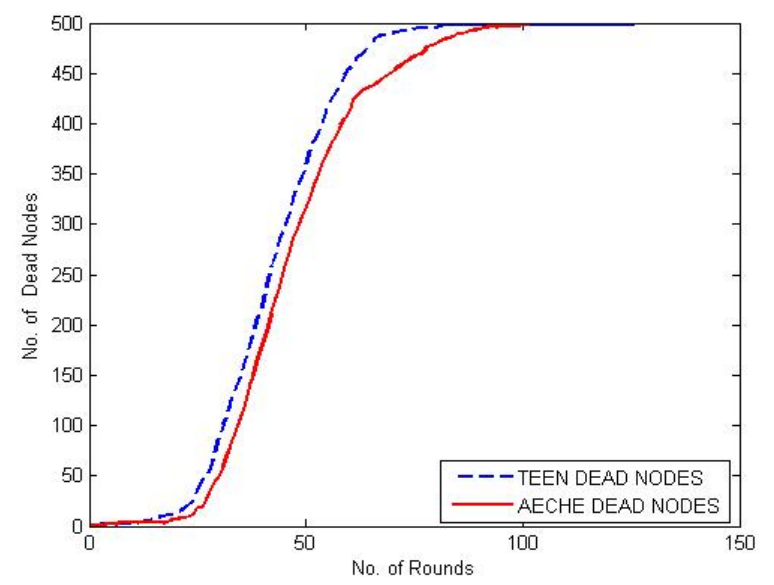

Figure 1. Network Life Time analysis between TEEN and AECHE

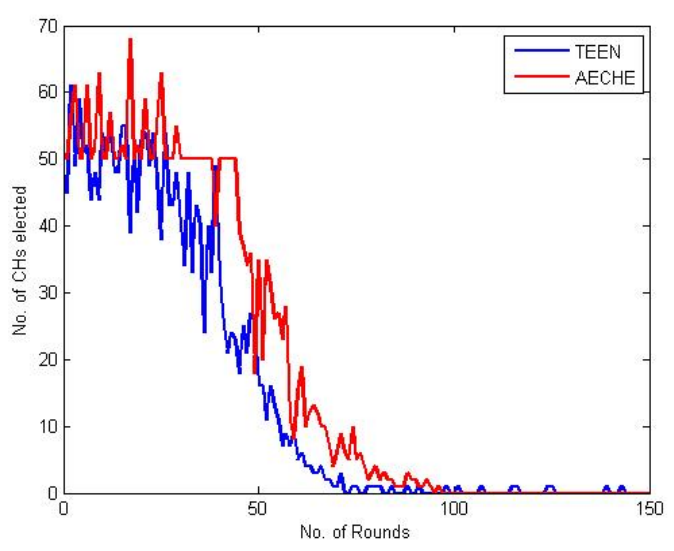

Figure 2. Number of clusters in various rounds 


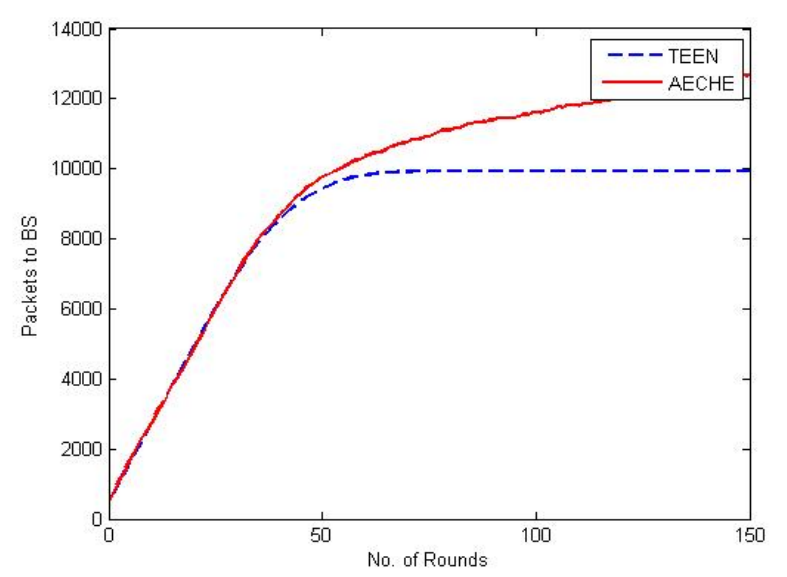

Figure 3. Packets received by Base Station using TEEN \& AECHE

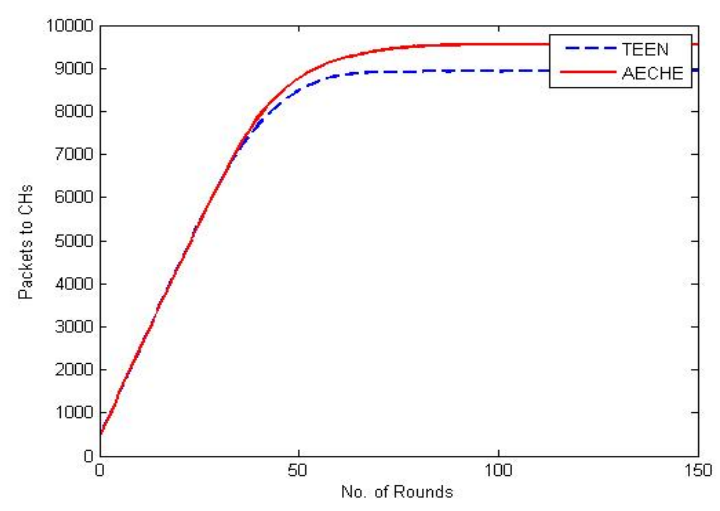

Figure 4. Packets received by Cluster heads using TEEN \& AECHE

In figure 3, it is analyzed the performance of TEEN and AECHE in terms packets sent to Base Station in the network. It indicates that AECHE performs better for the optimum number of clusters in the network. Figure 4 shows the number of packets sent to the cluster head by the nodes during the simulation period. Here also AECHE outperforms TEEN by a considerable margin. Therefore it is understood that AECHE has better throughput and network life time than TEEN.

\section{CONCLUSION}

In this paper, a novel technique called AECHE is proposed for the reactive sensor networks. The number of clusters for the reactive sensor networks will be optimally calculated in AECHE using the variation in active nodes and alive nodes between consecutive rounds. Therefore the energy of each node in the WSN is preserved and the network life is improved. This protocol balances the load between the active nodes by efficiently calculating the number of clusters. The simulation results based on the topology and network parameters show that the performance of the proposed protocol is improved in terms of throughput and network lifetime.

\section{REFERENCES}

1. Chen, Min, et al. "Body area networks: A survey." Mobile networks and applications 16.2 (2011): 171-193.
2. Zeng, Yuanyuan, et al. "Directional routing and scheduling for green vehicular delay tolerant networks." Wireless networks 19.2 (2013): 161-173.

3. Acampora, Giovanni, et al. "A survey on ambient intelligence in healthcare." Proceedings of the IEEE 101.12 (2013): 24702494.

4. Sheng, Zhengguo, et al. "A survey on the ietf protocol suite for the internet of things: Standards, challenges, and opportunities." Wireless Communications, IEEE 20.6 (2013): 91-98.

5. Akyildiz, Ian F., et al. "A survey on sensor networks." Communications magazine, IEEE 40.8 (2002): 102-114.

6. Estrin, Deborah, et al. "Next century challenges: Scalable coordination in sensor networks." Proceedings of the 5th annual ACM/IEEE international conference on Mobile computing and networking.ACM, 1999.

7. Cerpa, Alberto, et al. "Habitat monitoring: Application driver for wireless communications technology." ACM SIGCOMM Computer Communication Review 31.2 supplement (2001): 20-41.

8. Khedr, Ahmed M., and WalidOsamy. "Effective target tracking mechanism in a self-organizing wireless sensor network." Journal of Parallel and Distributed Computing 71.10 (2011): 1318-1326.

9. Nakamura, Eduardo F., Antonio AF Loureiro, and Alejandro C. Frery. "Information fusion for wireless sensor networks: Methods, models, and classifications." ACM Computing Surveys (CSUR) 39.3 (2007): 9.

10. Wei, Guiyi, et al. "Prediction-based data aggregation in wireless sensor networks: Combining grey model and Kalman Filter." Computer Communications 34.6 (2011): 793-802.

11. Rajagopalan, Ramesh, and PramodVarshney. "Dataaggregation techniques in sensor networks: a survey." IEEE Communications Surveys \& Tutorials 4.8 (2006): 48-63.

12. Smaragdakis, Georgios, Ibrahim Matta, and AzerBestavros. "SEP: A stable election protocol for clustered heterogeneous wireless sensor networks." Second international workshop on sensor and actor network protocols and applications (SANPA 2004).Vol. 3. 2004.

13. Xiang, Liu, Jun Luo, and Athanasios Vasilakos. "Compressed data aggregation for energy efficient wireless sensor networks." Sensor, mesh and ad hoc communications and networks (SECON), 2011 8th annual IEEE communications society conference on. IEEE, 2011.

14. Anastasi, Giuseppe, et al. "Energy conservation in wireless sensor networks: A survey." Ad hoc networks 7.3 (2009): 537-568.

15. Liu, Xuxun. "A survey on clustering routing protocols in wireless sensor networks." Sensors 12.8 (2012): 11113-11153.

16. Younis, Ossama, and Sonia Fahmy. "HEED: a hybrid, energyefficient, distributed clustering approach for ad hoc sensor networks." Mobile Computing, IEEE Transactions on 3.4 (2004): 366-379.

17. Chamam, Ali, and Samuel Pierre. "A distributed energyefficient clustering protocol for wireless sensor networks." Computers \& electrical engineering 36.2 (2010): 303-312.

18. Heinzelman, Wendi B., Anantha P. Chandrakasan, and HariBalakrishnan. "An application-specific protocol architecture for wireless microsensor networks." Wireless Communications, IEEE Transactions on 1.4 (2002): 660-670.

19. Sundareswaran, P., K. N. Vardharajulu, and R. S. Rajesh. "DECH: Equally Distributed Cluster Heads Technique for Clustering Protocols in WSNs." Wireless Personal Communications 84.1 (2015): 137-151.

20. Qing, Li, Qingxin Zhu, and Mingwen Wang. "Design of a distributed energy-efficient clustering algorithm for heterogeneous wireless sensor networks." Computer communications 29.12 (2006): 2230-2237.

21. Liu, Yuhua, Yongfeng Zhao, and JingjuGao. "A new clustering mechanism based on LEACH protocol." Artificial 
Intelligence, 2009.JCAI'09.International Joint Conference on.IEEE, 2009.

22. Lindsey, Stephanie, and Cauligi S. Raghavendra. "PEGASIS: Power-efficient gathering in sensor information systems." Aerospace conference proceedings, 2002.IEEE.Vol. 3.IEEE, 2002.

23. Manjeshwar, A., and D. P. Agrawal. "TEEN: a routing protocol for enhanced efficiency in wireless sensor networks."
Parallel and Distributed Processing Symposium., Proceedings 15th International. 2001.

24. Manjeshwar, Arati, and Dharma P. Agrawal. "APTEEN: A hybrid protocol for efficient routing and comprehensive information retrieval in wireless sensor networks." ipdps.IEEE, 2002. 